\title{
2015 Presidential Address-Society of Surgical Oncology: The Next 75 Years
}

\author{
Ronald J. Weigel, MD, PhD \\ Department of Surgery, University of Iowa, Iowa City, IA
}

It is an honor to stand before you as President of the Society of Surgical Oncology (SSO) to deliver this presidential address. We heard a great talk from Dr. Charles Balch about the history of the SSO during its first 75 years. My goal will be to outline the efforts underway to position the SSO to be successful in advancing its mission over the next 75 years.

Being President of the SSO is the pinnacle of my academic career in surgery and it is fitting for me to thank those individuals who have helped me and supported me throughout my academic career. First, I would like to thank my wife Paula and our four children, Gabriel, Carly, Isaac and John, whose daily presence has been yet another blessing throughout our life at the University of Iowa; Fig. 1a is a photo from the Iowa State Fair, where they sell deep-fried butter on a stick-we stuck to ice cream. I received tremendous help and scientific mentorship for my research training from Dr. George Miller who was my thesis advisor for my PhD work at Yale (Fig. 1b), and Dr. Joe Nevins with whom I spent a year as a post-doctoral fellow at Duke (Fig. 1c). These individuals have provided and continue to provide me with mentorship in my scientific career. I would like to acknowledge my surgery mentors, beginning with Dr. David Sabiston who was Chairman of the Department of Surgery at Duke during my training as a resident in surgery (Fig. 1d). I would also like to recognize Dr. John Niederhuber who gave me my first job and hired me as an Assistant Professor at Stanford University (Fig. 1e). Dr. Tim Eberlein has been ever-present throughout my surgical career, providing guidance,

(C) Society of Surgical Oncology 2015

First Received: 30 April 2015;

Published Online: 2 June 2015

R. J. Weigel, MD, PhD

e-mail: Ronald-Weigel@uiowa.edu advice and friendship that has helped me navigate through the tough times (Fig. 1f). Both Tim and Kim Eberlein have been steadfast friends to our family, as they have to countless other academic surgeons. We can never repay our mentors. The best we can do to show our gratitude is to pass it on and 'pay it forward'. I encourage all of you to pay it forward by supporting junior faculty in their development as surgical oncologists.

I would also like to recognize my fellow officers in the SSO: Dr. Jeff Drebin who has served as President-elect over the last year; Dr. Dan Coit, Vice President; Dr. Dave Bartlett, Treasurer; and Dr. Armando Giuliano, Secretary of the SSO (Fig. 1g-j). These individuals have been extremely supportive and dedicated to the Society throughout my time as an officer of the SSO. I would like to acknowledge several of the immediate past Presidents with whom I have had the honor of serving the SSO during their time as president, beginning with Dr. Bill Cance, Dr. Fabrizio Michelassi (who I also served with on the American Board of Surgery [ABS]), Dr. Mitch Posner (who is also currently President of the SSO Foundation), Dr. Jim Economou (who I have worked with in a variety of capacities, both in the SSO and in the American Surgical Association), Dr. Monica Morrow (who provided tremendous leadership and set the bar for leadership in the SSO during its transition), and Dr. Suzanne Klimberg (who I also served with on the ABS) (Fig. 1k-p). The quality and level of accomplishment and dedication of these individuals is unsurpassed. A special thanks and acknowledgment goes to Dr. Jim Howe, who has served as the Scientific Program Chair for the 2015 SSO Annual Meeting (Fig. 1q). He has done an absolutely fabulous job putting this meeting together here in Houston. These individuals have been instrumental in my journey to this pinnacle today and, not unlike the Sherpas who help on a mountain trek, I would not be standing here today without their help, advice, and heavy lifting. 
The SSO began its journey in 1940 as the James Ewing Society. Surgical science was characterized by a paper published by Dr. Alfred Blalock who noticed that partial occlusion of the renal artery in a dog induced hypertension. ${ }^{1}$ The classic paper from Dr. Whipple, describing a pancreaticoduodenectomy, characterized surgical care. ${ }^{2}$ At the time the James Ewing Society was founded, the operation now known as the Whipple Operation was actually performed in two stages and was associated with an operative mortality of $30 \%$. Basic science investigation was characterized by the work of Dr. Barbara McClintock, who I had the pleasure of seeing at Cold Spring Harbor when I was a graduate student at Yale. Dr. McClintock discovered that chromosomes are made up of a linear collection of genes, which determine phenotype. She also discovered transposons in maize in the 1940s, for which she won the Nobel Prize. ${ }^{3}$ The clinical application of basic discovery in 1940 was highlighted by the first mass production of penicillin. This was the state of our clinical and scientific endeavors 75 years ago. In the present state of surgical oncology, patients are diagnosed with computed tomography (CT) scans and magnetic resonance imaging (MRI) scans. Positron emission tomography (PET) scans are used to identify metastases and recurrences; operations are performed using laparoscopes and space-aged robotic surgical techniques. We have come a long way in a short time. But what is around the corner? What does the future hold? During this past year as President of the SSO, I have tried to position the Society to develop its role as a global leader in surgical oncology. This mission has led me on a trek across the globe. My year as President started in Phoenix at the Annual Meeting in 2014, then on to Iowa City, Chicago, Los Angeles, Chennai, India, San Francisco, Liverpool, New York, Philadelphia, Seoul, South Korea, and now ending here in Houston, Texas.

I would like to acknowledge the hard work of the staff of the SSO. These individuals, under the leadership of Eileen Widmer, provide support for your Society and allow us to fulfill the mission of the Society. One of my trips early in my presidential year was undertaken as a planning retreat to develop the future direction of the SSO and to determine how the SSO could best develop the future of surgical oncology as a profession. The focus of the SSO was developed as 'four pillars' (education, quality initiatives, research, and strategic relationships) designed to support the future of surgical oncology in the US and globally.
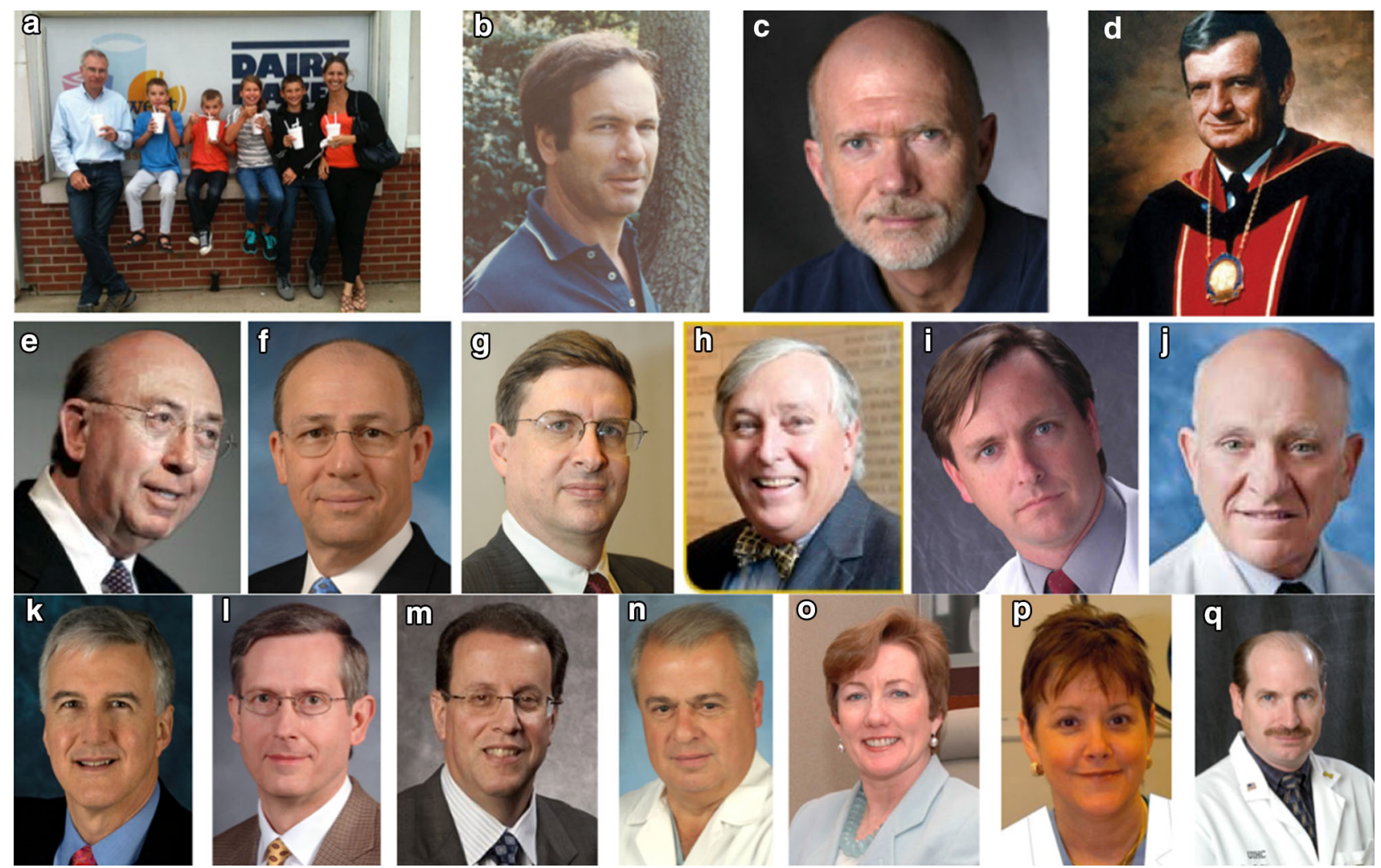

FIG. 1 Notable individuals and Society of Surgical Oncology leadership. a The Weigel Family, from left to right, Ron, Isaac, John, Carly, Gabriel, and Paula. b Dr. George Miller. c Dr. Joe Nevins. d Dr. David Sabiston. e Dr. John Niederhuber. f Dr. Tim

Eberlein. g Dr. Jeff Drebin. h Dr. Dan Coit. i Dr. Dave Bartlett. j Dr. Armando Giuliano. k Dr. Bill Cance. I Dr. Fabrizio Michelassi. m Dr. Mitch Posner. n Dr. Jim Economou. o Dr. Monica Morrow. p Dr. Suzanne Klimberg; and q Dr. Jim Howe 


\section{SOCIETY OF SURGICAL ONCOLOGY (SSO) EDUCATIONAL PROGRAMS}

Education in Surgical Oncology is a key mission of the SSO. The SSO Training Committee under the leadership of the former chair, Dr. Russell Berman, and current chair, Dr. Kevin Roggin, has supported the educational programs of our Society. I am pleased to note that we now have Board certification in surgical oncology with a new certificate of added qualifications in Complex General Surgical Oncology. The principles of Surgical Oncology Fellowship Training have remained dedicated to education in multidisciplinary care, the surgical management of complex cancer care, education in clinical trial design and research, community outreach, patient counseling, including the appropriate and timely use of palliative care, and leadership in oncology. ${ }^{4}$

The ABS provided approval for Complex General Surgical Oncology in 2011 and, as a director of the ABS at the time, I was honored to work with former SSO PresidentsDr. Michelassi and Dr. Klimberg - in moving this initiative forward. ${ }^{5}$ The ABS created a new surgical oncology subboard of the ABS, and programs are now accredited by the Accreditation Council for Graduate Medical Education (ACGME). As with ABS certification of candidates in surgery, Complex General Surgical Oncology candidates must pass a written qualifying examination and an oral certifying examination. The inaugural Complex General Surgical Oncology certifying examination was held in Philadelphia from 9 to 11 February of this year (Fig. 2). Individuals were tested in five areas-breast, hepaticopancreaticobiliary, melanoma-sarcoma, endocrinestomach, and colorectal. There were a total of 63 examinees, and now 56 diplomats, who are board certified in Complex General Surgical Oncology, representing a pass rate of $89 \%$. This is an historic moment for the SSO and the development of surgical oncology nationally. It is the culmination of work by countless individuals over decades and I am honored that this historic success occurred during my presidency. Boardcertified surgical oncologists will form the basis of surgical oncology care of the future.

The Breast Oncology Fellowship is another highlight of our educational program. There are now 42 SSO-approved breast oncology programs that have been developed in collaboration with the American Society of Breast Surgeons (ASBS) and the American Society of Breast Disease. We also have six new applications for additional programs. This is a 1-year multidisciplinary training program with a new curriculum, which is being rolled out in July 2015. There are strict requirements, as well as a site visit by the SSO Training Committee. I am further pleased to note that during the past year the SSO was awarded Accreditation with Commendation from the Accreditation Council for Continuing Medical Education (ACCME) for our educational programs. A note of recognition goes out to Dr. Danny Takanishi, the former Chair of the SSO Continuing Medical Education (CME) Committee, and Dr. Glenda Callender, who is the current CME Committee Chair. This accomplishment would not have been possible without their dedication. The CME Committee is responsible for all SSO CME activities and the accreditation with commendation demonstrates the exceptional content of the SSO CME offerings and the robust efforts to assure that our programs are free of bias and conflicts of interest.

Many new additional educational programs are now in place and being developed to educate future surgical oncologists. The Fellows Institute continues to be an important and valuable educational resource for Surgical Oncology and Breast Oncology Fellows. This program is co-sponsored by the SSO, the ASBS, and the Americas Hepato-Pancreato-Biliary Association (AHPBA). The Surgical Oncology Self-Assessment Program (SOSAP)

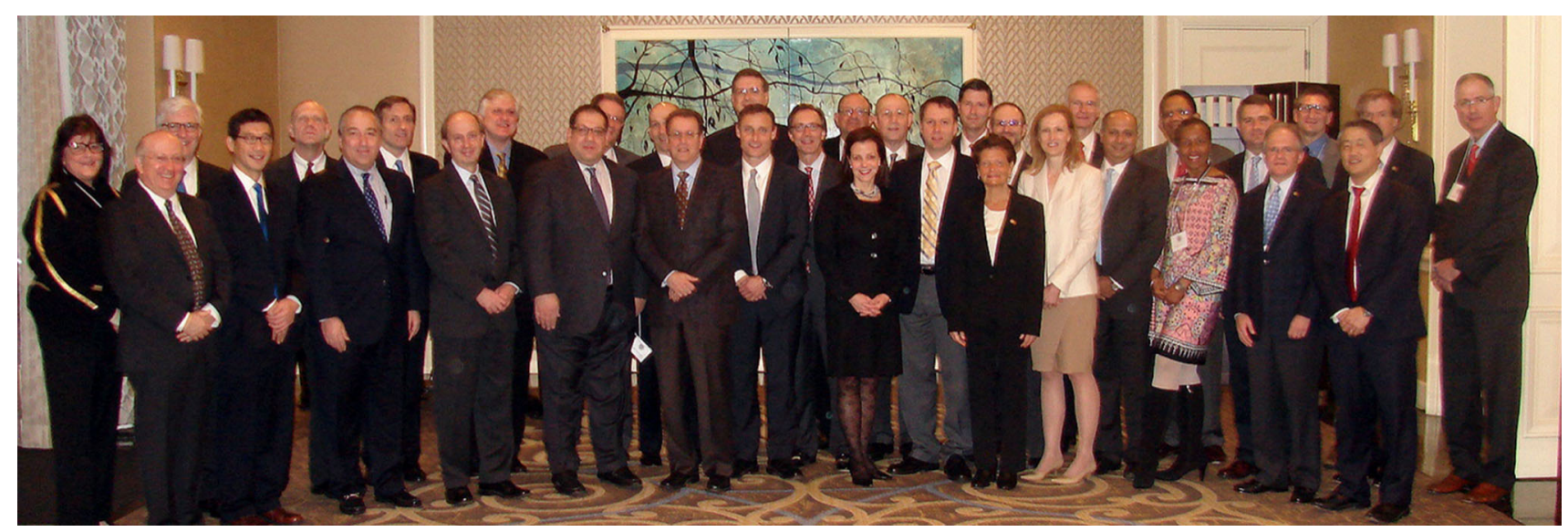

FIG. 2 Inaugural CGSO examiners. Group photo of examiners for the inaugural Complex General Surgical Oncology Certifying Examination, Philadelphia, 9-11 February 2015 

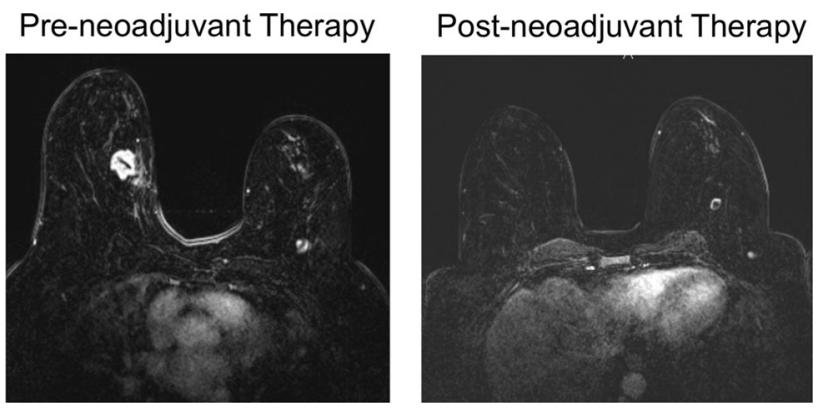

FIG. 3 MRI of locally advanced breast cancer. Pre- and postneoadjuvant chemotherapy MRI scan of patient with locally advanced breast cancer demonstrates complete pathologic response. MRI magnetic resonance imaging

was developed to provide a comprehensive review course for fellows preparing for the qualifying examination in Complex General Surgical Oncology. SOSAP is also a valuable resource for $\mathrm{CME}$ and MOC for general surgeons whose practices largely involve oncology. The SCORE project is being led by Dr. Russ Berman in conjunction with the ABS to develop a teaching curriculum for surgical oncology trainees in surgical residency and fellowships. The virtual meeting and Best of SSO allow local and international members to have access to the educational programs of the SSO annual meeting. These programs are being expanded globally to our international partners. Annals of Surgical Oncology will continue to be an important forum for dissemination of cutting-edge research and reviews. The journal also recently started a new CME program offering self-assessment MOC credits. In addition, we are nearing completion of an expanded 10-year contract with Springer, with whom we hope to develop other unique products in surgical oncology.

\section{SSO QUALITY INITIATIVES}

The next important area we have focused on are quality initiatives. The strategic group in Quality has been led by Dr. Sandra Wong, with major contributions from Dr. Sharon Weber, Dr. Jonathan Zager, Dr. Ned Carp, and Dr. Doug Tyler. The goal of the Quality Committee is to promote high-quality, cost-effective, multidisciplinary cancer care for surgical patients. In order to accomplish this, we have developed a Quality Workgroup. These individuals are involved in developing surgical quality metrics in conjunction with the Commission on Cancer, the oncology National Surgical Quality Improvement Program (NSQIP) National Cancer Institute (NCI) Center Consortium, as well as the National Comprehensive Cancer Network. In addition, the SSO has sponsored the publication and endorsement of patient-care pathways. Examples of these important position papers include the recent publication in Annals of Surgical Oncology on breast cancer margins, ${ }^{6}$ and an upcoming review on neoadjuvant therapy in breast cancer. These two examples have been developed under the leadership of the Past-President, Dr. Monica Morrow. Ongoing development of quality metrics in melanoma and gastrointestinal cancer are also being proposed.

\section{SSO RESEARCH}

Research needs to play an expanded role of the SSO moving forward. The strategic plan for research in the SSO was led by Dr. Jeff Drebin, and has been taken over by Dr. Rich Alexander, as Chair of the newly formed Research Committee. The goal of the SSO research mission is to foster basic, translational, and clinical cancer research. This is accomplished through educational symposia and lectures designed to aid SSO members in developing research projects and preparing successful research grants. Facilitating the interaction of appropriate mentorship needs to be an expanded activity of the SSO. The SSO has also funded awards; there is the ongoing Clinical Investigator Award (CIA), which is an award given by the SSO for US $\$ 100,000$. There is a new initiative to develop seed grants, as well as more substantive grants, to support basic and translational science. The SSO Foundation will play an important role in fundraising to continue the SSO grant funding mechanisms.

\section{SSO STRATEGIC RELATIONSHIPS}

We have expanded our strategic relationships with the goal of leveraging the expertise in the SSO to facilitate the development of surgical oncology and advance the mission of the SSO. Our partnerships with corporations will play an increasing role in the SSO working with our members and the SSO Foundation, which was re-branded this year from the James Ewing Foundation. However, we recognize that strategic partnerships with corporations may involve a conflict of interest, but we are moving forward in a way that the SSO can advance its mission through collaboration with companies to advance research and clinical care. Many of these companies have embraced the goal of improving patient care in oncology and this is clearly an area in which we can work together.

The SSO has worked collaboratively with other societies in several ways. As one example, the SSO was involved in a joint training conference involving the AHPBA and American Society of Transplant Surgeons (ASTS). Dr. Rohan Jeyarajah organized the conference during the ACS in San Francisco. The SSO representatives included Dr. Berman and Dr. Mitch Posner. There was also an opportunity for me to meet with the AHPBA President, Dr. Elijah Dixon, and ASTS President, Dr. Peter Stock, to 
discuss other ways for our societies to work together to advance training and clinical care. Other collaborative discussions are underway with the AHPBA, ASBS, and the American Association of Endocrine Surgeons to offer joint symposia and potentially coordinate meeting locations and times.

Several years ago the SSO Executive Council approved a plan to expand our relationships with international surgical societies. We now have formal memorandums of understanding (MOUs) with the European Society of Surgical Oncology, the Indian Association of Surgical Oncology, the Brazilian Society of Surgical Oncology, the Mexican Society of Oncology, and the Japanese Society of Gastroenterological Surgery. These important international relationships have expanded the scope of the SSO to becoming a global society and in the near future are likely to include the Korean Society of Surgical Oncology and the newly formed Latin American Society of Surgical Oncology. The MOUs developed with these international partners have developed new faculty exchange programs, joint symposia and plenary sessions at our annual meetings, International Career Development Exchange programs, and Best of SSO for presentations at their annual and regional meetings. Many individuals have fostered the development of international relationships by the SSO but special recognition goes to Dr. Balch, Dr. Coit, Dr. Posner, Dr. Leon, and Dr. Anderson. Expanding these international relationships are a critical part of the SSO having greater recognition as a global surgical oncology leader.

\section{THE FUTURE OF SURGICAL ONCOLOGY}

These new initiatives by the SSO have been developed with our view on the close horizon, but what lies beyond the immediate short-term future? What is the future of surgical oncology? What does the future of surgical oncology look like? In what direction should the SSO move to prepare for that future? To answer this question, consider for a moment breast cancer, which is a common disease treated by surgical oncologists. Fig. 3 is an example of a pre- and post-chemotherapy MRI scan in a woman who had locally advanced breast cancer and was treated with neoadjuvant chemotherapy. She had a complete response by imaging and was found to have had a complete pathologic response. What will be the role of surgical oncologists when chemotherapy or other targeted therapy results in an increasing number of patients who develop a complete pathologic response? The answer to this question is that the role of surgical oncologists needs to extend beyond operative therapy alone.

As another example of the changing landscape in oncology care, consider another cancer that is commonly treated by surgical oncologists-melanoma. There have been tremendous strides made in understanding the biology of melanoma. New biologic insights are being discovered with the identification of pathways, which drive the growth and progression of melanoma. Based on these pathways, new drugs are coming online which specifically target key drivers of melanoma growth and progression. There has been an explosion in our understanding of tumor immunology and the complex immune response involving tumor antigens, $\mathrm{T}$ cells, and dendritic antigen-presenting cells. From these findings, multiple clinical trials are being developed, which are based on a better understanding of the immunologic basis of melanoma, and with impressive results.

Surgical oncologists of the future will be board-certified, will have to be trained in multidisciplinary care, and will have an in-depth understanding of cancer biology (Fig. 4). They will need the training to allow them to effectively function as the 'team leader'. There will be a greater emphasis on preventative and palliative care. As we move away from 'fee-for-service' reimbursement models towards population health, preventative and palliative care will play a greater role in oncology care. Personalized medicine will play a greater role. Genetic information about the patients, as well as their tumors, will be used to direct therapy. Molecular and cellular analysis will inform pharmacogenomics to be able to specifically target cancer treatment in an individual patient.

Directed cancer therapy requires a greater understanding of the biologic characteristic of cancers. Cancer patients who were once thought to have a single, homogeneous disease are being stratified based on molecular profiling of the tumor, identifying distinct groups of patients based on molecular markers, which are predictive of prognosis and response to therapy (Fig. 5). Patient-specific drug sensitivities and resistance, based on individual genetic differences, will be used to avoid adverse drug effects. After stratification, molecular and genetic profiling will be

\section{Surgical Oncology of the Future}

\author{
- Board Certified in Complex General Surgical \\ Oncology \\ - Need to "train for the future" \\ - Greater emphasis on preventative and \\ palliative care \\ - Personalized Medicine \\ - Greater use of genetic information and \\ molecular characterization \\ - Expansion of pharmacogenomics
}

FIG. 4 Surgical oncology of the future. Key predictions of surgical oncology care in the future 


\section{Personalized CancerTherapy}

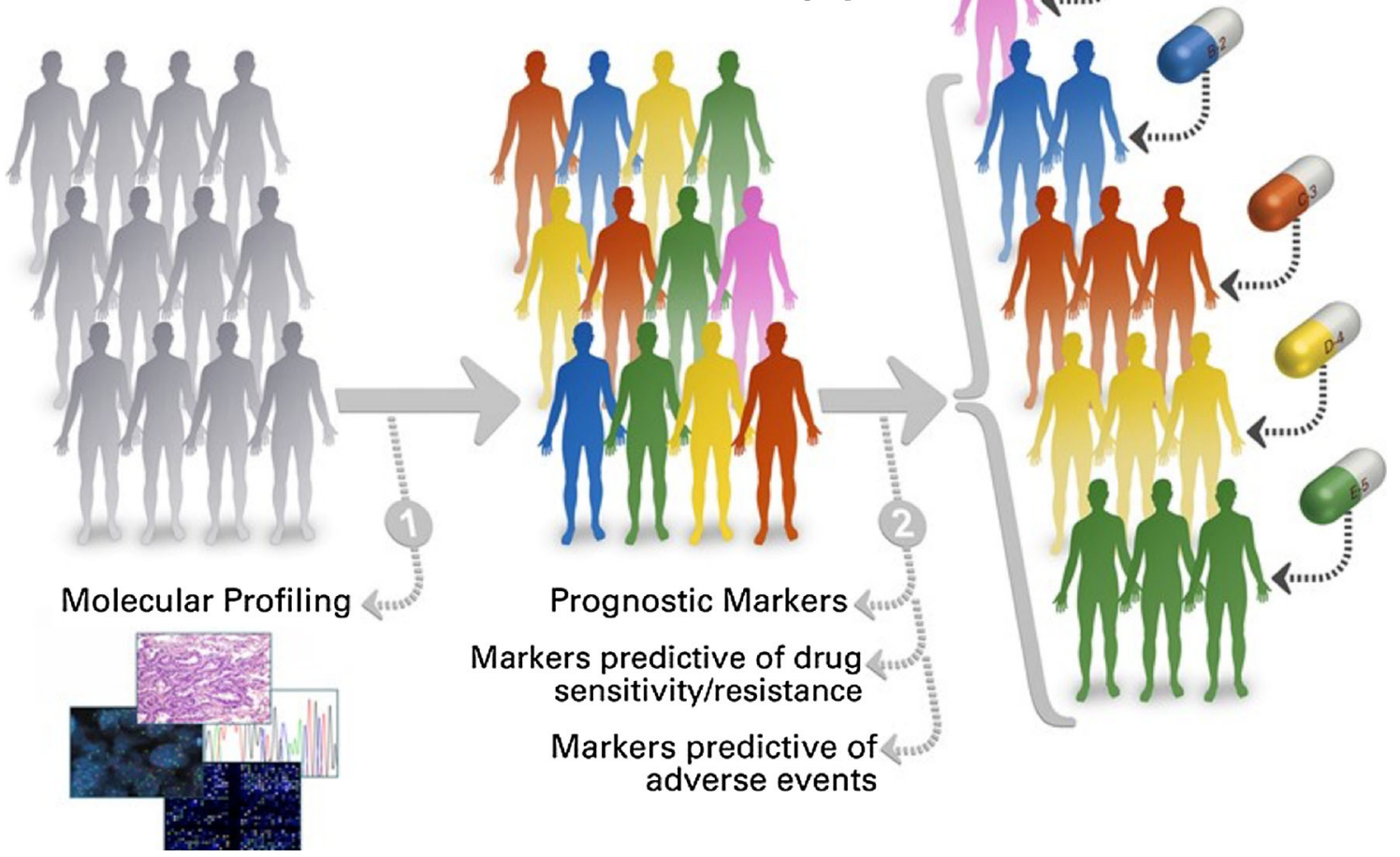

FIG. 5 Personalized cancer therapy. Diagram showing how molecular profiling of tumors help to statify patients into defined molecular categories, allowing for targeted therapy based on molecular markers,

used to direct specific treatments, which will provide greater effectiveness with a lower chance of complications.

What role will surgical oncologists play in the new era of personalized therapy? There are several concerning issues related to trends for research and development in the US. As one example, scientific research and development spending from 2012 to 2013 decreased significantly in the US and Canada, while many other countries have increased their investment in research (Fig. 6a). This is one piece of evidence that we are falling behind in research as a nation compared with our global partners. An analysis of NIH spending over the past few decades reveals a significant decrease in NIH funding in real dollars over the past 10 years (Fig. 6b). Both total $\mathrm{NIH}$ dollars and total funding of R01 equivalent grants has decreased consistently. Although one might say that US\$20 billion is a lot of money, compare the NIH budget to military spending. The national defense budget is in the range of US\$600-700 billion (Fig. 6c). Just the funding for Iraq and Afghanistan alone dwarfs the NIH budget, and only a fraction of the NIH budget is dedicated to the war on cancer, a war against an enemy that is just as scary and just as lethal and much closer to home as the ones we fight overseas. We have to make choices as a nation. The SSO needs to have a clear position and voice its support for cancer research. which can predict response. From: "Personalized Cancer Therapy" website, https://pct.mdanderson.org/, last accessed May 1, 2015

The outlook for research funding for surgeons is particularly concerning. Data from a paper published in Annals of Surgery show that NIH awards to academic surgeons is declining relative to their nonsurgical colleagues (Fig. 6d). ${ }^{7}$ The total medical school surgical faculty over this time period was noted to increase by $132 \%$; however, NIH awards to surgical faculty decreased by $47 \%$. In addition, it was noted there was a growing gap between awards for surgical and non-surgical faculty. Sadly, the research career of the majority of surgeon-scientists is short, with only $20 \%$ engaged in research by 59 years of age.

So, what can the SSO do? First, we need to place a greater emphasis on research in residency and fellowship training. We need to partner with the NCI to strengthen the role of surgical oncology as a recognized scientific field. Surgeon-scientists bring a unique perspective to oncology care and we have a fundamental role in oncology research. We need to advocate for more funding for cancer research, particularly solid organ cancers treated by surgical oncologists. We need to increase the recruitment of students who have an interest in basic cancer biology and who will be capable of advancing scientific discovery in surgical oncology. Finally, you can make a difference. One easy thing everyone here can do is to support the SSO 


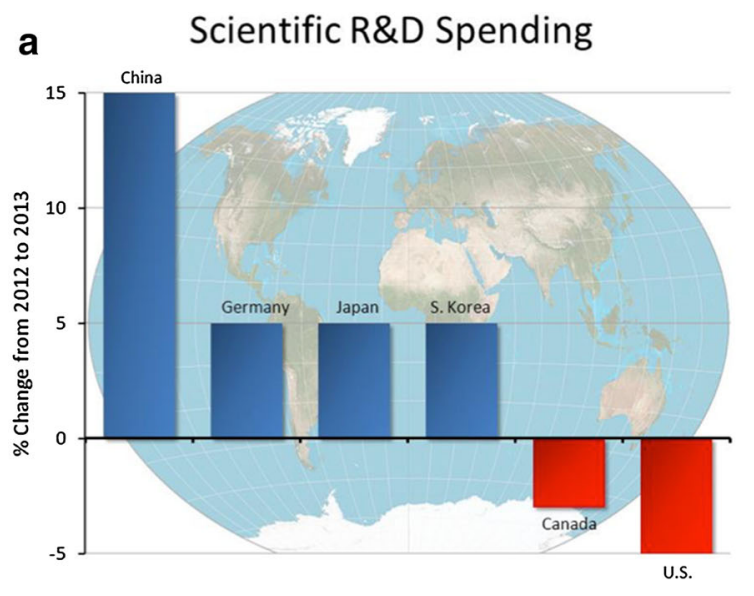

Source: Cell. 2013 Jul 3;154(1):16-9.

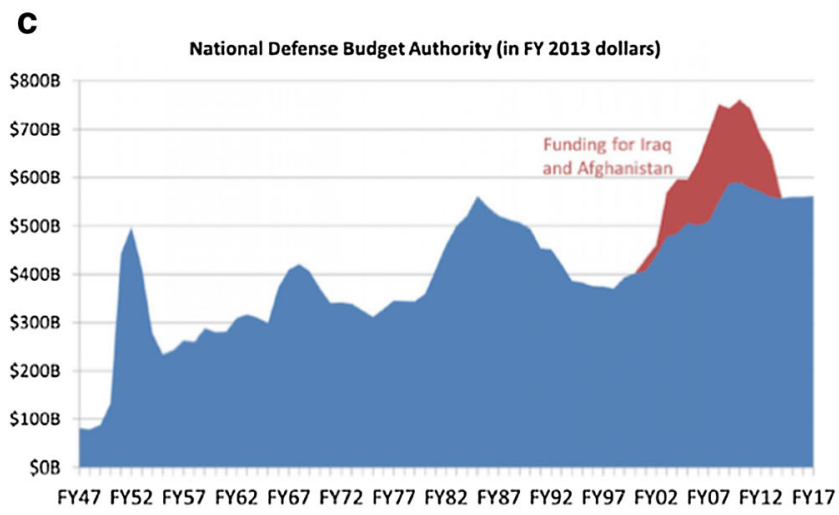

FIG. 6 Funding trends. a Scientific R\&D spending for the US and comparison countries; from: http://directorsblog.nih.gov/2013/09/24/ one-nation-in-support-of-biomedical-research/, last accessed May 1, 2015. b Trends in NIH funding from 1992 to 2013; from: http://www. brimr.org/NIH_Awards/Trends/, last accessed May 1, 2015. c National

Foundation, which provides educational and research funding for young surgical oncologists.

The surgical oncologist of the future will no doubt need to remain a surgeon, but he or she will also need to be a geneticist, a molecular biologist, a bioinformaticist, a pharmacologist, and a radiologist. In order to accomplish this task, we need to train the best and the brightest in surgical oncology. Surgical oncology has a brilliant future, if we are willing to evolve beyond operative therapy of the cancer patient. We need to embrace the future, and the SSO needs to create the tools and structure to prepare the next generation to be successful surgical oncologists of the future. It has been a tremendous honor to serve as your president. I thank the leadership of the SSO for the opportunity to serve you as the 2015 SSO President.

CONFLICT OF INTEREST The author declares that he has no conflict of interest. b
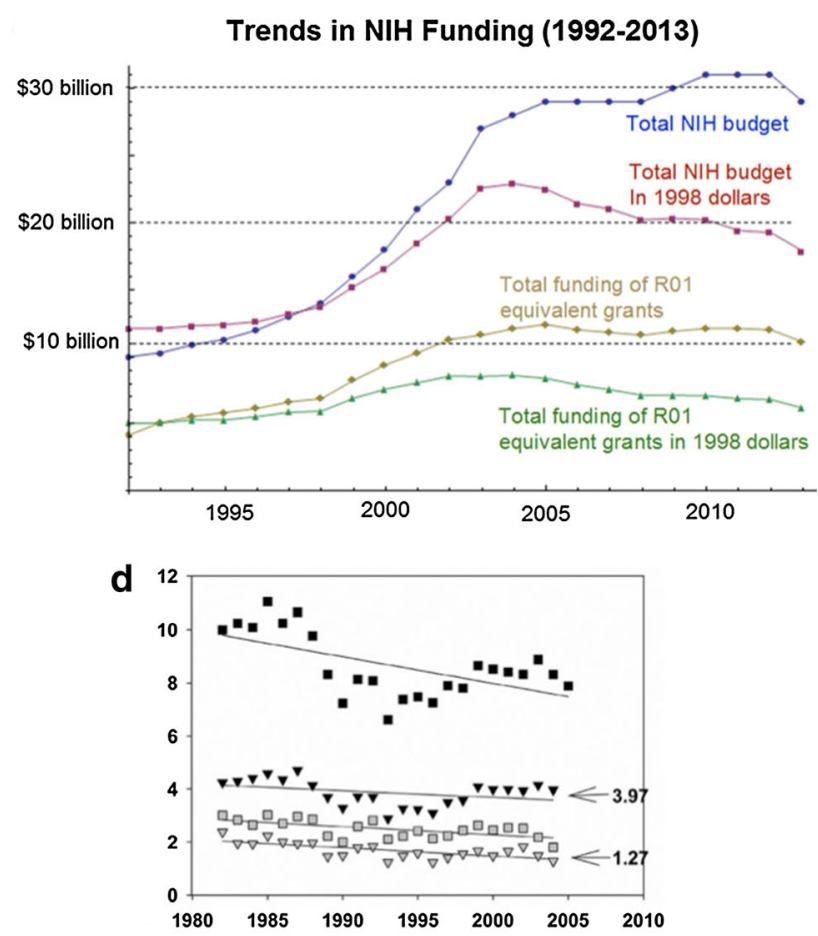

$$
\begin{aligned}
& \text { - Total NIH awards } \\
& \text { NIH awards to faculty with an MD } \\
& \text { NIH awards to surgical faculty } \\
& \nabla \quad \text { NIH awards to surgical faculty wih an MD }
\end{aligned}
$$

defense spending since 1947; from: http://www.decisionsonevidence. com/2013/01/federal-spending-problems-follow-the-money/nationaldefense-budget-authority-in-fy2013-dollars/, last accessed May 1, 2015; d Awards to surgical faculty, Reprinted with permission from Mann et al.

\section{REFERENCES}

1. Blalock A, Levy SE, Cressman RD. Experimental hypertension: the effects of unilateral renal ischemia combined with intestinal ischemia on the arterial blood pressure. J Exp Med. 1939;69(6):833-46.

2. Whipple AO. The rationale of radical surgery for cancer of the pancreas and ampullary region. Ann Surg. 1941;114(4):612-5.

3. McClintock B. The significance of responses of the genome to challenge. Science. 1984;226(4676):792-801.

4. Berman RS, Weigel RJ. Training and certification of the surgical oncologist. Chin Clin Oncol. 2014;3(4):45.

5. Michelassi F. 2010 SSO presidential address: subspecialty certificate in advanced surgical oncology. Ann Surg Oncol. 2010; 17(12):3094-103.

6. Moran MS, Schnitt SJ, Giuliano AE, et al. Society of Surgical Oncology-American Society for Radiation Oncology consensus guideline on margins for breast-conserving surgery with wholebreast irradiation in stages I and II invasive breast cancer. Ann Surg Oncol. 2014;21(3):704-16.

7. Mann M, Tendulkar A, Birger N, Howard C, Ratcliffe MB. National Institutes of Health funding for surgical research. Ann Surg. 2008;247(2):217-21. 